\title{
Diagnostik und Therapie asymptomatischer Nebennierentumoren
}

\author{
M. Reincke, W. Winkelmann, C. Jaursch-Hancke, D. Kaulen, J. Nieke, G. Ollenschläger und B. Allolio \\ Medizinische Universitätsklinik II (Direktor: Prof. Dr. W. Kaufmann), Krankenhaus Köln-Merheim
}

Bei 23 Frauen und neun Männern im mittleren Alter von 54 (25-73) Jahren wurde bei der Klärung anderer Beschwerden zufällig ein asymptomatischer Nebennierentumor entdeckt. In allen Fällen ließen sich die Tumoren computertomographisch darstellen. Achtmal waren sie beidseits lokalisiert, in je 12 Fällen rechts- oder linksseitig. Der durchschnittliche Tumordurchmesser betrug $3(1-9) \mathrm{cm}$. Vier Tumoren $(12,5 \%$ ) wiesen eine endokrine Aktivität auf (ein Phäochromozytom, drei cortisolproduzierende Tumoren). Acht Patienten wurden adrenalektomiert, dabei ergaben sich sechs Nebennierenadenome, ein benignes Phäochromozytom und ein Ganglioneurom. Eine Feinnadelbiopsie wurde bei zwei Patienten vorgenommen, der zytologische Befund war benigne. Computertomographische Verlaufskontrollen bei elf $(34,4 \%)$ der nicht-operierten Patienten 6-48 Monate (im Mittel 14 Monate) später zeigten bei keinem der Patienten eine Größenzunahme des Tumors. Daher erscheint es bei zufällig diagnostizierten Nebennierentumoren gerechtfertigt, zunächst einmal den Verlauf zu beobachten, da gutartige Prozesse offensichtlich weitaus häufiger sind als maligne. Bei einem Tumordurchmesser von mehr als $6 \mathrm{~cm}$ ist jedoch wegen des Malignitätsrisikos eine Adrenalektomie durchzuführen.

\section{The diagnosis and treatment of asymptomatic adrenal tumours}

Adrenal tumours had been discovered incidentally (since 1981) in 32 patients (23 females and nine males; mean age 54 [25-73] years) who had had computed tomography (CT) or ultrasonography for other reasons: none had a history or symptoms of such tumour. Tumours were bilateral in eight, right or left-sided in 12 each: all had been confirmed by CT. Average tumour size was $3 \mathrm{~cm}(1-9 \mathrm{~cm})$. Three patients had cortisol-producing adrenal tumours, and there was one benign phaeochromocytoma (abnormally high adrenaline and noradrenaline excretion). Fine-needle biopsies in two patients revealed a benign histology. An adrenalectomy was performed in eight patients (the one phaeochromocytoma, six adenomas and one ganglioneuroma). Follow-up CT in 11 of the non-operated patients 6-48 months later (mean of 14 months) did not demonstrate any increase in tumour size so that a waiting attitude seems justified: benign tumours are clearly much more frequent than malignant ones. However, if the tumour diameter is greater than $6 \mathrm{~cm}$, an adrenalectomy is indicated because of the danger of malignancy.
In der Vergangenheit wurden Nebennierentumoren zumeist diagnostiziert, wenn ein entsprechender klinischer Befund auf eine Hormonüberproduktion hinwies oder die Tumoren durch ihre Größe lokale Beschwerden verursachten. Seit der Einführung von bildgebenden Verfahren wie Sonographie und Computertomographie in die Routinediagnostik werden Nebennierenraumforderungen jedoch in zunehmenden Maße zufällig diagnostiziert (1-6, 8, 11, 12, 14, 16), bevor klinische Hinweise auf einen hormonell aktiven oder inaktiven Nebennierentumor auftreten. Prognose und Therapie dieser Nebennierentumoren sind noch umstritten. Die therapeutischen Empfehlungen gehen zum Teil weit auseinander. Einerseits wird wegen des Malignitätsrisikos zur Operation (14), andererseits aber auch primär zu Verlaufsbeobachtungen geraten (2), da das Nebennierenkarzinom ein äußerst seltener Tumor ist.

Im folgenden berichten wir über $32 \mathrm{~Pa}$ tienten, die sich zur Klärung eines zufällig diagnostizierten Nebennierentumors in unserer Klinik vorstellten. 


\section{Patienten und Methoden}

Die Daten aller Patienten mit der Diagnose $\gg \mathrm{Ne}-$ bennierentumor «, die ab 1981 in unserer Klinik behandelt worden waren, wurden retrospektiv ausgewertet. Als Zufallsbefund wurde diejenige Nebennierenraumforderung definiert, bei der kein Zusammenhang zwischen der Indikation zur Sonographie oder Computertomographie und dem Nebennierentumor bestand und sich anamnestisch und klinisch kein Anhalt für einen Nebennierentumor ergab.

41 Patienten, die von uns ambulant oder stationär behandelt worden waren, erfüllten diese Kriterien. Bei sieben Patienten wurde die Diagnose primär sonographisch gestellt, sie wurde jedoch anschließend computertomographisch gesichert. Dabei erwiesen sich alle Raumforderungen morphologisch als solide, während zystische Raumforderungen nicht beobachtet wurden. Neun Patienten wurden wegen unzureichender Dokumentation der Untersuchungsergebnisse und (oder) unzureichender Diagnostik von der Studie ausgeschlossen. Von den verbleibenden 32 Patienten waren $23(72 \%)$ weiblich und neun $(28 \%)$ männlich. Die Indikationen zur Computertomographie gibt Tabelle 1 wieder.

Tab. 1 Indikationen zur Computertomographie

\begin{tabular}{l|c|c}
\hline \multirow{2}{*}{ Indikation } & \multicolumn{2}{|l}{ Patienten } \\
& $n$ & $\%$ \\
\hline Oberbauchschmerzen & 8 & 25,0 \\
Nebennierentumor im Ultraschall & 7 & 21,8 \\
Hypertoniediagnostik & 4 & 12,5 \\
- langjähriger essentieller arterieller Hypertonus & 3 & \\
- therapeutisch schwer beeinfluBbarer essentieller & & \\
$\quad$ arterieller Hypertonus & 1 & \\
Verdacht auf Pankreasmalignom & 3 & 9,4 \\
Nierenerkrankung & 2 & 6,3 \\
idiopathischer Hirsutismus & 2 & 6,3 \\
andere & 6 & 18,7 \\
\hline Summe & 32 & 100 \\
\hline
\end{tabular}

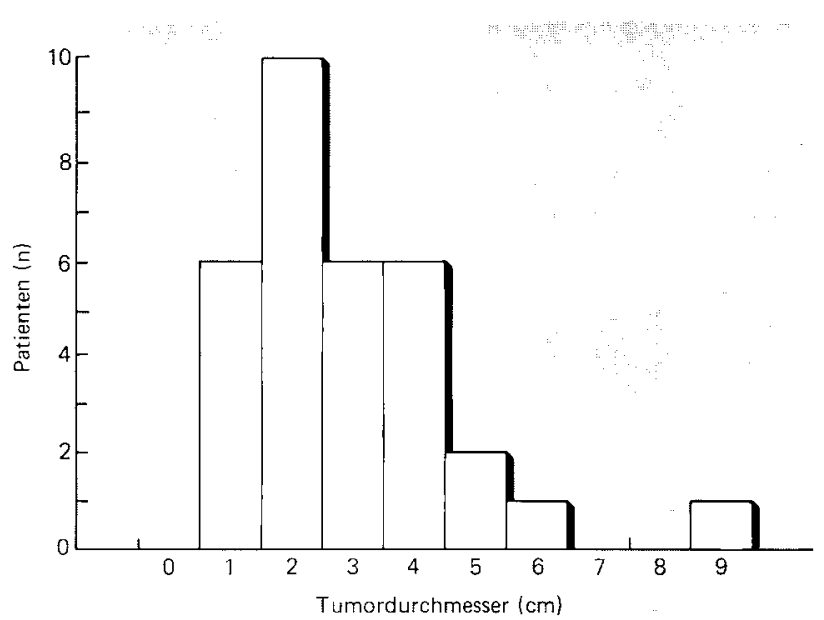

Abb. 1 Maximaler computertomographisch bestimmter Nebennierentumordurchmesser bei 32 Patienten mit zufällig diagnostizierten Nebennierentumoren.
Bei jeweils zwölf Patienten $(37,5 \%)$ bestand ein rechts- bzw. linksseitiger Nebennierentumor. Acht Patienten $(25 \%)$ wiesen beidseitige Nebennierentumoren auf. Der durchschnittliche Tumordurchmesser lag bei $3,0 \mathrm{~cm}$ (Bereich: 1,0 bis $9,0 \mathrm{~cm}$, Abbildung 1).

Das Alter der Patienten lag im Mittel zum Zeitpunkt der Diagnose bei 54 Jahren (Bereich: 25 bis 73 Jahre). Die Altersverteilung zeigte einen eingipfligen Kurvenverlauf mit einem Altersgipfel bei 60 bis 64 Jahren. Auffällige klinische Befunde waren eine Adipositas in $56 \%$, ein essentieller arterieller Hypertonus in $53 \%$ und ein Diabetes mellitus Typ II in 16\% der Fälle (Tabelle 2).

Tab. 2 Klinische Befunde bei Patienten mit zufällig diagnostizierten Nebennierentumoren (Mehrfachnennungen)

\begin{tabular}{l|r|l}
\hline \multirow{2}{*}{ Befunde } & \multicolumn{2}{|l}{ Patienten } \\
& $\mathrm{n}$ & $\%$ \\
\hline Adipositas & 18 & 56,3 \\
(essentieller) arterieller Hypertonus & 17 & 53,1 \\
Diabetes mellitus Typ II & 6 & 18,8 \\
generalisierte Arteriosklerose & 5 & 15,6 \\
Malignom-Anamnese & 1 & 3,1 \\
\hline
\end{tabular}

Die endokrine Diagnostik umfaßte routinemäßig neben umfangreichen Aufnahmeuntersuchungen einen niedrig dosierten Dexamethason-Hemmtest (3 mg Dexamethason um 23 Uhr, Bestimmung von Serum-Cortisol um 9 Uhr) sowie die Bestimmung von Aldosteron, Adrenalin und Noradrenalin im 24-Stunden-Sammelurin. Die Serum-Cortisolkonzentrationen (NEN, Dreieich) und die Aldosteronkonzentrationen im Urin (Serono, Freiburg) wurden radioimmunologisch bestimmt. Die Adrenalin- und Noradrenalinkonzentrationen im Urin wurden anhand der Fluoreszenz-Spektralphotometrie mit einer Modifikation der von Lund (10) beschriebenen Methode ermittelt.

\section{Ergebnisse}

Ein pathologischer DexamethasonHemmtest (Serum-Cortisol $>5 \mu \mathrm{g} / \mathrm{dl}$ um $9 \mathrm{Uhr}$ ) fand sich bei sechs Patienten. Die weitere Diagnostik ergab bei drei dieser Patienten eine unauffällige Regulation der Cortisolsekretion. Drei Patienten wiesen cortisolproduzierende Nebennierentumoren auf, ohne daß klinisch Hinweise auf ein Cushing-Syndrom bestanden (Tabelle 3). Bei zwei Patienten lag nach endokrinologischen Kriterien das Vollbild eines Cushing-Syndroms vor (pathologischer Dexamethason-Hemmtest, aufgehobene Tagesrhythmik der Cortisolsekretion, erhöhte Ausscheidung von Cortisol im 24-Stunden-Urin). Bei dem dritten Patienten bestand dagegen die seltene Konstellation eines »Prä-Cushing-Syndroms«, mit noch erhaltener Tagesrhythmik bei pathologischem Dexamethason-Hemmtest und leicht erhöhter Ausscheidung von Cortisol im 24-Stunden-Urin. Bei allen drei Patienten wurde eine Adrenalektomie durchgeführt und ein Nebennierenadenom entfernt.

Eine pathologisch erhöhte Adrenalin( $>10 \mu \mathrm{g})$ und Noradrenalausscheidung (> 100 $\mu \mathrm{g}$ ) im 24-Stunden-Urin bestand nur bei einer Patientin mit 
Tab. 3 Klinische, histologische und zytologische Befunde bei acht Patienten mit Adrenalektomie und zwei Patienten mit Feinnadelbiopsie

\begin{tabular}{c|l|l|l|l|l|l}
\hline Fall & Geschlecht & $\begin{array}{l}\text { Alter } \\
\text { (Jahre) }\end{array}$ & $\begin{array}{l}\text { Tumor- } \\
\text { durchmesser } \\
\text { (cm) }\end{array}$ & endokrine Aktivität & Histologie & Hypertonus \\
\hline \multicolumn{2}{c|}{ Adrenalektomie } & & & & & \\
1 & weiblich & 54 & 9,0 & Adrenalin- und Noradrenalinsekretion & benignes Phäochromozytom & - \\
2 & männlich & 40 & 6,0 & cortisolproduzierender Tumor & Nebennierenrindenadenom & - \\
3 & weiblich & 64 & 4,5 & cortisolproduzierender Tumor & Nebennierenrindenadenom & - \\
4 & weiblich & 37 & 4,0 & cortisolproduzierender Tumor & Nebennierenrindenadenom & postoperativ persistierend \\
5 & weiblich & 41 & 6,0 & - & Nebennierenrindenadenom & postoperativ persistierend \\
6 & weiblich & 62 & 5,0 & - & Nebennierenrindenadenom & - \\
7 & mannnlich & 25 & 4,0 & - & Ganglioneurom & - \\
8 & weiblich & 60 & 1,2 & - & Nebennierenrindenadenom & postoperativ persistierend \\
\hline Feinnadelbiopsie & & & - & Nebennierenadenomzellen & - \\
9 & weiblich & 59 & 2,5 & - & Nebennierenadenomzellen & - \\
10 & weiblich & 61 & 2,3 & - &
\end{tabular}

einem Nebennierentumor von $9 \mathrm{~cm}$ im Durchmesser. Histologisch fand sich bei der Adrenalektomie ein benignes Phäochromozytom.

Eine erhöhte Aldosteron-Exkretion im 24-Stunden-Urin wiesen insgesamt sieben Patienten auf $(21,9 \%)$. Bei allen konnte ein primärer Hyperaldosteronismus (Conn-Syndrom) als Ursache ausgeschlossen werden.

Tabelle 3 gibt eine Übersicht über die histologischen Befunde der acht adrenalektomierten Patienten. Überwiegend fanden sich Nebennierenrindenadenome $(n=6)$. Eine mittels Sonographie oder Computertomographie gesteuerte Nebennierenpunktion wurde bei zwei weiteren Patienten vorgenommen. Die zytologischen Befunde waren benigne.

Computertomographische Verlaufskontrollen bei elf Patienten $(34,4 \%)$ über im Mittel 14 Monate (Bereich: 6 bis 48 Monate) zeigten bisher keine signifikante Größenzunahme der Nebennierentumoren.

\section{Diskussion}

Von der Nebenniere ausgehende Tumoren galten lange Zeit als sehr selten. Erst in den sechziger Jahren konnte in mehreren Sektionsstudien gezeigt werden, daß sich bei nicht ausgewählten Patienten Nebennierentumoren, die zu Lebzeiten keine Symptomatik hervorgerufen hatten, in einer Häufigkeit von 1,4 bis $8,7 \%$ fanden $(7,9,15)$. Zumeist handelte es sich hierbei um relativ kleine Nebennierenadenome, deren Durchmesser bei 0,5 bis $6,0 \mathrm{~cm}$ (Mittel: $1,0 \mathrm{~cm}$ ) lag. Deshalb ist es nicht verwunderlich, daß seit Einführung der Computertomographie in die klinische Routine, mit der heute eine Darstellung der Nebennierenregion in über $95 \%$ der Fälle gelingt (5), in zunehmender Häufigkeit Nebennierenraumforderungen als Zufallsbefunde diagnostiziert werden $(1-6,8,11,12,14,16)$. Schätzungen zufolge werden solche Nebennierenraumforderungen bei 0,5 bis $0,7 \%$ aller abdominellen Computer- tomographien gefunden $(1,3)$. Unsere eigenen Erfahrungen bestätigen die Beobachtung, daß es sich hierbei im allgemeinen um gutartige, sehr langsam wachsende Nebennierentumoren handelt. Weder zeigte sich bei computertomographischen Verlaufskontrollen, die bei $34 \%$ unserer Patienten zur Verfügung standen, ein signifikantes Größenwachstum, das auf einen rasch wachsenden malignen Tumor hingedeutet hätte, noch fand sich bei den adrenalektomierten oder biopsierten Patienten (zusammen $31 \%$ ) ein maligner Nebennierentumor. Einschränkend muß jedoch betont werden, daß $35 \%$ der Patienten nicht in unserer Klinik weiterbetreut wurden, so daß über ihren weiteren Krankheitsverlauf keine Daten vorliegen.

Der vergleichsweise hohe Prozentsatz endokrin aktiver Tumoren bei unseren Patienten $(12,5 \%)$ belegt die Bedeutung einer sorgfältigen endokrinen Diagnostik. Neben der Bestimmung von SerumElektrolyten wird deshalb in unserer Klinik ein Dexamethason-Hemmtest durchgeführt und die Konzentrationen von Aldosteron und Katecholaminen im 24-Stunden-Urin bestimmt. Hierdurch wird eine frühzeitige Diagnose des asymptomatischen Phäochromozytoms und damit die Senkung der Morbidität und Letalität dieser Erkrankung ermöglicht. Aber auch bei cortisolproduzierenden Nebennierentumoren ist eine Diagnose im asymptomatischen Stadium von Vorteil, weil durch eine frühzeitige Operation die zum Teil irreversiblen Folgeerkrankungen, wie Osteoporose, Diabetes mellitus und Arteriosklerose, verhindert werden können.

Das Vorgehen bei asymptomatischen, nicht endokrin aktiven Nebennierenraumforderungen ist zur Zeit noch umstritten. Copeland (2) stellte 1983 in einem Übersichtsartikel die Befunde von 51 Patienten mit zufällig diagnostizierten Nebennierentumoren zusammen. 15 Patienten wiesen Nebennierenmetastasen eines bekannten Primärtumors auf, bei 36 Patienten lag ein gutartiger Nebennierenbefund vor. Hieraus folgerte er, da $\beta$ bis zu einem Durchmesser von $6 \mathrm{~cm}$ bei Patienten ohne Malignom-Anamnese keine chirur- 
gische Intervention nötig sei, da das Risiko eines Nebennierenkarzinoms vernachlässigbar klein sei. Hingegen geben Seddon und Mitarbeiter (14) primär einem chirurgischen Vorgehen den Vorzug. Sie beobachteten bei 11 Patienten mit zufällig diagnostiziertem Nebennierentumor drei Nebennierenkarzinome und ein Neuroblastom. Offensichtlich handelte es sich jedoch nicht um eine repräsentative Auswahl von Patienten, da die überwiegende Anzahl der Tumoren vor Einführung der Computertomographie mittels konventioneller radiologischer Verfahren (Abdomen-Leeraufnahme, intravenöse Pyelographie) diagnostiziert wurden und somit ungewöhnlich groß waren. Belldegrun und Mitarbeiter (1) beschrieben 1986 bei insgesamt 28 Patienten 17 gutartige Veränderungen (Durchmesser $1-6 \mathrm{~cm}$ ), fünf Nebennierenkarzinome (Durchmesser $6-15 \mathrm{~cm}$ ) und sechs Nebennierenmetastasen bisher nicht diagnostizierter Primärtumoren (Durchmesser $1-10 \mathrm{~cm}$ ). Im Gegensatz zu Copeland empfehlen sie eine explorative Laparotomie ab einem Tumordurchmesser von $3,5 \mathrm{~cm}$.

Die Ergebnisse unserer Studie entsprechen eher den Resultaten von Copeland. Da das Nebennierenrindenkarzinom ein sehr seltener Tumor ist (Inzidenz 1:1700000 pro Jahr) und der Tumordurchmesser bei Diagnose meist mehr als $6 \mathrm{~cm}$ beträgt (13), raten wir bei zufällig diagnostizierten, endokrin inaktiven Nebennierentumoren mit einem Durchmesser von weniger als $6 \mathrm{~cm}$ zu engmaschigen computertomographischen Verlaufskontrollen, bei größeren Tumoren oder bei Wachstumstendenz zur Adrenalektomie. Unseres Erachtens kann durch dieses Vorgehen das Risiko, ein Nebennierenrindenkarzinom zu übersehen, sehr gering gehalten werden. Gleichzeitig wird hierdurch vielen Patienten eine unnötige Laparotomie erspart.

Eine mittels Ultraschalls oder Computertomographie gesteuerte Feinnadelbiopsie ist nach unseren Erfahrungen zur Klärung der Dignität eines Nebennierentumors wenig geeignet, da adenomatöse von karzinomatösen Nebennierenveränderungen zytologisch nur schwer abgegrenzt werden können (2). Daher trägt die Biopsie wenig zur Entscheidungsfindung bei und wird von uns nur in seltenen Fällen durchgeführt. Eine Ausnahme sind zystische Nebennierenveränderungen, bei denen die Zytologie in einem hohen Prozentsatz die Diagnose ermöglicht.

Da zufällig diagnostizierten Nebennierenraumforderungen auch Metastasen eines noch nicht erkannten Primärtumors zugrunde liegen können, ist die Suche nach einem Primärtumor bei der Erstuntersuchung erforderlich. In Anlehnung an Copeland (2) empfehlen wir das in Abbildung 2 dargestellte Vorgehen.

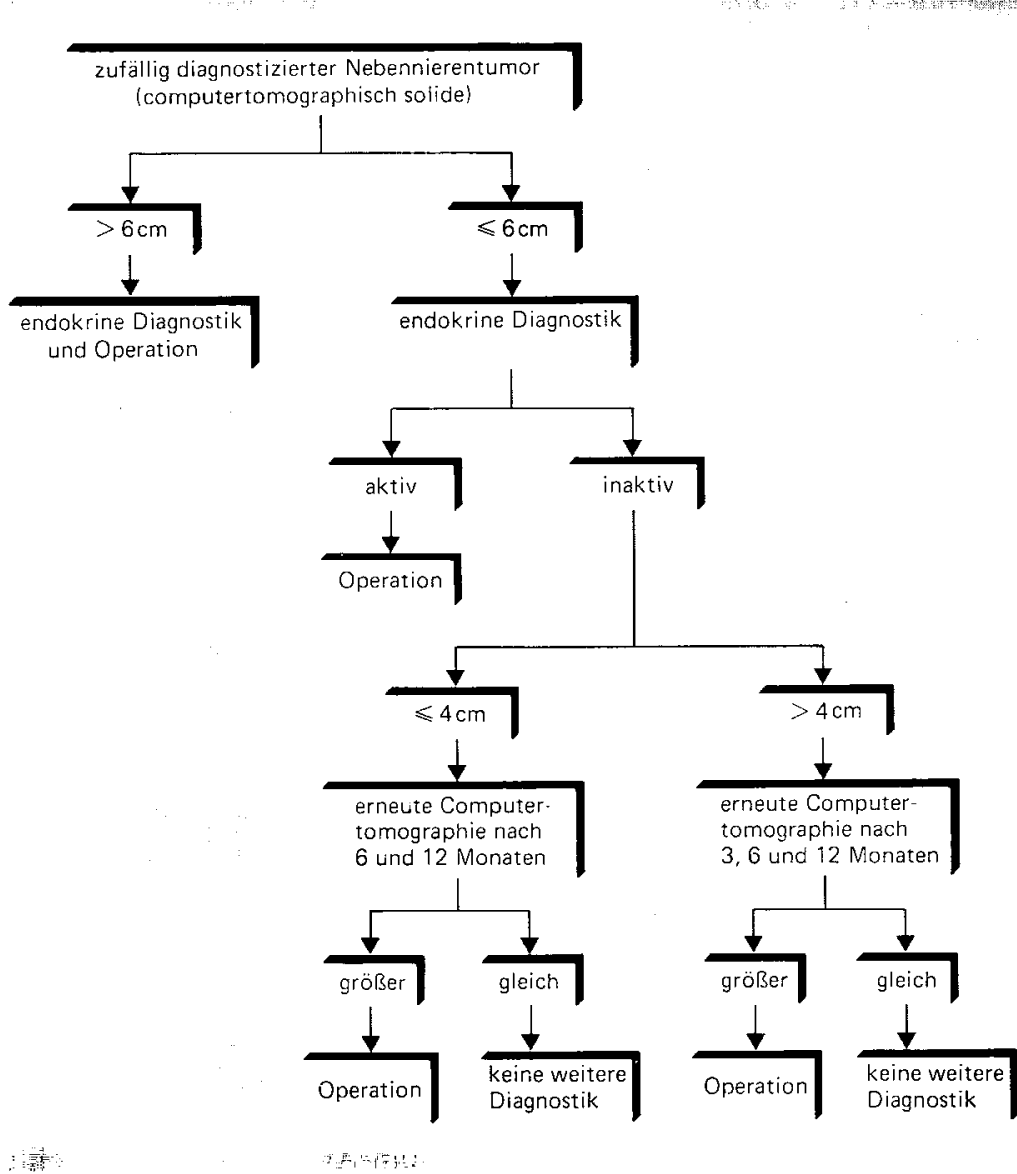

Abb. 2 Diagnostisches und therapeutisches Vorgehen bei Patienten mit asymptomatischen, soliden Nebennierentumoren, modifiziert nach Copeland (2) 
Ein interessanter Aspekt bei zufällig diagnostizierten Nebennierentumoren ist die auffällige Häufigkeit eines gleichzeitig bestehenden arteriellen Hypertonus, bei unseren Patienten in $53 \%$ der Fälle. Dabei bestand der endokrinen Diagnostik zufolge bei allen Patienten ein essentieller arterieller Hypertonus. Bei zwei Patienten mit arteriellem Hypertonus und endokrin inaktivem Nebennierenadenom kam es postoperativ nicht zu einer Normalisierung der Blutdruckwerte. Offensichtlich spielen somit asymptomatische Nebennierenadenome keine Rolle in der Pathogenese des Hypertonus. Das gehäufte Auftreten eines Hypertonus bei diesen Patienten könnte damit erklärt werden, daß Hypertoniepatienten, ebenso wie Patienten mit Adipositas oder fortgeschrittener Arteriosklerose, aufgrund von Sekundärerkrankungen häufiger computertomographisch untersucht werden. Dagegen spricht jedoch, daß in mehreren Sektionsstudien ebenfalls eine positive Korrelation zwischen arteriellem Hypertonus und $\mathrm{zu}$ Lebzeiten nicht erkannten Nebennierenadenomen gefunden wurde $(7,15)$. Eine klare Antwort auf diese Fragen kann jedoch eine retrospektive Studie nicht geben.

\section{Literatur}

1 Belldegrun, A., S. Hussain, S. E. Seltzer, K. R. Loughlin, R. F. Gittes, J. P. Richie: Incidentally discovered mass of the adrenal gland. Surg. Gynec. Obstet. 163 (1986), 203.

2 Copeland, P. M.: The incidentally discovered adrenal mass. Ann. intern. Med. 98 (1983), 940.

3 Garz, G., M. Lüning, B. Melzer: Computertomographische Zufallsbefunde von hormoninaktiven Nebennierenrindenadenomen Radiol. diagn. (Berl.) 26 (1985), 761.

4 Geelhoed, G. W., E. M. Druy: Management of the adrenal sincidentaloma«. Surgery 92 (1982), 866.

5 Glazer, H. S., P. J. Weyman, S. S. Sagel, R. G. Levitt, B. L. McClennan: Nonfunctioning adrenal masses. Amer. J. Radiol. 139 (1982), 81.

6 Guerrero, L. A.: Diagnostic and therapeutic approach to incidental adrenal mass. Urology 26 (1985), 435.

7 Hedeland, H., G. Östberg. B. Hökfelt: On the prevalence of adrenocortical adenomas in an autopsy material in relation to hypertension and diabetes. Acta med. scand. 184 (1986), 211.

8 Katz, R. L., A. Shirkhoda: Diagnostic approach to incidental adrenal nodules in the cancer patient. Cancer (Philad.) 55 (1985), 1995.

9 Kokko, J. P., T. C. Brown, M. M. Bermann: Adrenal adenoma and hypertension. Lancet $1967 /$ I, 468 .

10 Lund, A.: Fluorimetric determination of adrenalin in blood. Acta pharmacol. 5 (1949), 75.

11 Mitnick, J. S., M. A. Bosniak, A. J. Megibow, D. P. Naidich: Nonfunctioning adrenal adenomas discovered incidentally on computed tomography. Radiology 148 (1983), 495.

12 Prinz, R. A., M. H. Brooks, R. Churchill, J. L. Graner, A. M Lawrence, E. Paloyan, M. Sparagana: Incidental asymptomatic adrenal masses detected by computed tomography scanning. J. Amer. med. Ass. 248 (1982), 701.

13 Richie, J. P., R. F. Gittes: Carcinoma of the adrenal cortex. Cancer (Philad.) 45 (1980), 1957.

14 Seddon, J. M., N. Baranetsky, P. J. van Boxel: Adrenal wincidentalomas«. Urology 25 (1985), 1.

15 Shamma, A. H., J. W. Goddard, S. C. Sommers: A study of the adrenal status in hypertension. J. chron. Dis. 8 (1985), 587.

16 Waldner, H., D. Wilker, B. Eibl-Eibesfeld: Therapeutisches Vorgehen beim »Incidentalom« der Nebenniere. Chirurg 57 (1986), 557.

Dr. M. Reincke, Prof. Dr. W. Winkelmann,

Dr. Cornelia Jausch-Hancke, Dr. D. Kaulen,

Dr.J. Nieke, Dr. G. Ollenschläger.

Privatdozent Dr. B. Allolio

Medizinische Universitätsklinik II

Städtisches Krankenhaus Merheim

Ostmerheimer Str. 200

D-5000 Köln 91 